\title{
Bougie-assisted cricothyroidotomy: Delphi-derived essential steps for the novice learner
}

\author{
Alia Dharamsi, MD*; Sara Gray, MD, MPH ${ }^{* \neq}$; Christopher Hicks, MD, MEd**; \\ Jonathan Sherbino, MD, MEd ${ }^{\S \pi}$; Melissa McGowan, $\mathrm{MKS}^{\ddagger}$; Andrew Petrosoniak, MD, MSc*
}

\author{
CLINICIAN'S CAPSULE \\ What is known about the topic? \\ The steps for performing a bougie-assisted cricothyroi- \\ dotomy (BAC) have not been systematically developed, \\ leading to instructional and performance variability. \\ What did this study ask? \\ Can the modified Delphi process be used to establish the \\ essential steps required for BAC instruction? \\ What did this study find? \\ Using a modified Delphi methodology, we developed a \\ 17-item BAC checklist to guide instruction for novice \\ learners. \\ Why does this study matter to clinicians? \\ This checklist, developed by expert consensus, can be \\ used to improve consistency in training for this life- \\ saving procedure.
}

\section{ABSTRACT}

Objective: A cricothyroidotomy is a life-saving procedure, performed as a final option to emergency airway algorithms, and is essential for all clinicians who perform emergency airway management. The bougie-assisted cricothyroidotomy (BAC) is a novel technique that may be performed faster and with fewer complications than other traditional approaches. There is no established standard set of steps to guide the instruction of BAC performance. This study sought to systematically develop a BAC checklist for novice instruction using a modified Delphi methodology and international airway experts. Methods: A literature review informed the creation of a preliminary BAC checklist. A three round, modified Delphi method was used to establish a BAC checklist intended for novice-level instruction. The consensus level for each step and the final checklist were predefined at $80 \%$. Participants were international airway experts identified by study personnel and snowball sampling.

Results: Fourteen international airway experts across six acute care specialities participated in the study. The checklist was refined using a seven-point rating scale for each item and participant comments. A 17-item checklist was developed with expert consensus achieved after three rounds. Internal consistency, measured with Cronbach's $\alpha$, was $0.855(95 \%$ confidence interval 0.73-0.94).

Conclusion: This modified Delphi-derived checklist is the first systematically developed list of essential steps for guiding BAC instruction for novice learners. This tool serves to standardize BAC skill instruction and provide learners with a structured and consistent set of steps for deliberate practice.

\section{RÉSUMÉ}

Introduction: La cricothyroïdotomie est une intervention de sauvetage de dernier recours d'après les algorithmes d'assistance respiratoire d'urgence et que doivent connaître tous les cliniciens qui pratiquent le maintien de la perméabilité des voies respiratoires. La cricothyroïdotomie par bougie (CB) est une nouvelle technique qui peut se pratiquer plus rapidement et entraîner moins de complications que les techniques habituelles d'intervention. Toutefois, il n'existe pas de procédure établie, présentée en différentes étapes, sur la manière d'exécuter la $C B$. L'étude visait donc à élaborer, de manière structurée, une liste de vérification en vue d'une $C B$, conçue à l'intention des débutants, à l'aide d'une version modifiée de la méthode Delphi et d'experts en matière de maintien de la perméabilité des voies respiratoires, provenant de différents pays.

Méthode: Un examen de la documentation a permis tout d'abord de dresser une liste préliminaire de vérification en vue d'une $C B$. Par la suite, une liste de vérification conçue à l'intention des débutants a été élaborée à l'aide d'une version modifiée de la méthode Delphi, après trois tours. Le degré de consensus à atteindre à chaque étape et pour la liste de vérification définitive a été préétabli à $80 \%$. Les participants étaient des experts en matière de maintien de la perméabilité des voies respiratoires, provenant de différents pays, qui ont été trouvés par du personnel de recherche et selon la technique du sondage en boule de neige.

From the *Division of Emergency Medicine, Department of Medicine; Interdepartmental Division of Critical Care, University of Toronto, Toronto, ON; ‡Department of Emergency Medicine, St. Michael's Hospital, Toronto, ON; §Division of Emergency Medicine, Department of Medicine, McMaster University, Hamilton, ON; and IProgram for Education Research and Development, McMaster University, Hamilton, ON.

Correspondence to: Dr. Andrew Petrosoniak, St. Michael's Hospital, Department of Emergency Medicine, 30 Bond Street, Toronto ON M5B 1W8; Email: petro82@gmail.com 
Résultats: Quatorze experts, pratiquant dans six champs de spécialité en soins actifs, ont ainsi participé à l'étude. La liste de vérification a par la suite été précisée à l'aide d'une échelle d'évaluation à 7 points pour chacun des éléments et pour les remarques des participants. Une liste de vérification en 17 points a finalement été dressée après trois tours de recherche de consensus entre les experts. La consistance interne, mesurée selon le coefficient alpha de Cronbach, s'est établie à 0,855 (intervalle de confiance à $95 \%$ : 0,73-0,94).
Conclusion: II s'agit de la première liste de vérification des étapes essentielles à l'exécution de la $\mathrm{CB}$, qui a été dressée de manière structurée à l'aide de la méthode Delphi et conçue à I'intention des débutants. L'outil sert à uniformiser l'enseignement de la $\mathrm{CB}$ et offre aux apprenants une démarche structurée et consistante en vue de la pratique intentionnelle.

Keywords: airway, education/methods, education/teaching, performance improvement, qualitative research

\section{INTRODUCTION}

A cricothyroidotomy is a life-saving procedure, performed as a final option to emergency airway algorithms. ${ }^{1}$ This skill is essential for all clinicians who perform emergency airway management. However, it is only required for an estimated $0.2 \%$ of emergency tracheal intubations. ${ }^{2}$ The lack of clinical opportunities presents a challenge for procedural skill acquisition and maintenance. ${ }^{3-6}$

There are a few well described methods to perform a cricothyroidotomy comprising either open or percutaneous techniques. Recently, a novel technique termed bougie-assisted cricothyroidotomy (BAC) was described. ${ }^{1}$ Several features make this technique especially appealing during time-sensitive situations, including familiar and accessible equipment and fewer manoeuvres compared with other methods. While there are no head-to-head clinical trials comparing BAC with other techniques, simulation-based comparative studies suggest higher success rates, fewer complications, and faster completion compared with other traditional techniques. ${ }^{1,7-9}$ As a result, BAC is increasingly popular within the emergency medicine (EM) community and is the recommended surgical technique in the 2015 Difficult Airway Society (DAS) guidelines. ${ }^{1}$

Deconstructing a complex task into a series of integrated steps may be helpful for the novice learner. ${ }^{10}$ Procedural checklists can form the basis of instruction, framing, and standardizing the essential steps for the learner. Checklists have been successfully used to train novices on procedures like lumbar punctures, arterial lines, and central lines. ${ }^{11-16}$

To our knowledge, the steps involved in performing a safe and successful BAC have never been fully described, leading to variability in how the procedure is taught to novices. The modified Delphi process is a widely used method to establish consensus among experts where the evidence is incomplete or uncertain. ${ }^{17}$ The benefit of a
Delphi process for developing a rare procedure checklist is that expert opinion and experience can be collected, collated, and systematically analysed. ${ }^{17}$ The objective of this study was to develop a list of essential steps required for BAC performance by, and instruction of, novices using a modified Delphi process.

\section{METHODS}

\section{Study design}

The study design followed previously published methods ${ }^{11-16}$ to derive a set of essential steps for procedural skill performance, outlined in Figure 1.

\section{Checklist development}

The study team consisted of EM physicians at two academic, tertiary care trauma centres, in Toronto and Hamilton, Ontario. An English language literature search using multiple variations of the key words "checklist," "cricothyroidotomy," "cricothyrotomy," "bougie-assisted," "bougie," "surgical airway" on the PubMed, Ovid/Medline databases yielded 18 articles. None of the articles described a formal checklist development process for BAC performance. Each listed step for BAC performance was extracted and amalgamated into a common list. Duplicate steps were removed to form the first-iteration checklist as part of the modified Delphi process. This checklist contained 22 steps.

\section{Expert recruitment}

Each member of the study team submitted a list of physician airway management experts for study participation. Expert participants were selected based on a combination of their clinical, educational, and research experience in emergency airway management 


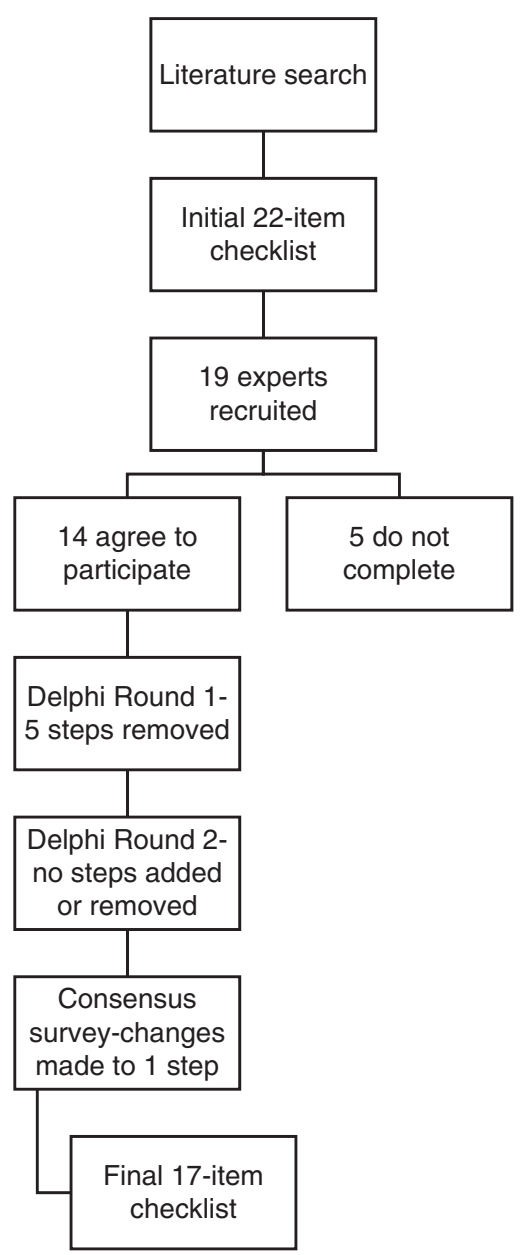

Figure 1. Overview of Study.

and knowledge of BAC, and were contacted via email. Snowball sampling among participants was used to expand the pool of participants from the 12 initially emailed, to 18 total. $^{18}$ There is no consensus on the number of participants to perform a Delphi $;{ }^{17}$ however, previous Delphi-derived checklists for procedural skills included 10-12 participants. ${ }^{11-16}$ In this study, 14 physician experts agreed to participate (Table 1). Experts were blinded to the identity of other Delphi group members for the entire study, and participant responses were de-identified before the data analysis.

\section{Delphi process}

The modified Delphi process followed published methods used to derive procedural skill checklists. ${ }^{11-17}$ Three rounds were completed over 6 months to develop a refined and consensus-based checklist. Each participant was contacted by the study coordinator who also exclusively received all survey responses. All data were de-identified prior to a review and analysis. Throughout the process, all participants were blinded to the identity and specific comments of the other study participants; participants received summarized comments during each round to mitigate any bias that may have been associated with others' feedback. In Round 1, participants received the 22-item checklist developed from existing literature. They scored each step on a scale of 1-7, where 1 represented "not important at all" and 7 indicated "an egregious error if missed." Participants were asked to suggest additional steps as needed, propose wording changes to existing steps, and add free text comments to further qualify their rankings. After Round 1, participant comments were reviewed and consolidated. Consistent with methodologies from modified Delphi studies for other procedural skills, the mean for each step was calculated, and steps with a mean $\leq 3.0$ were removed for the next round. ${ }^{11-16}$ The revised checklist was electronically sent to the individual reviewers with a summary of the comments from the previous round. In Round 2, the process was repeated using the checklist version developed from the preceding round. The checklist was finalized in the third round, and consensus was achieved for each step and the list in its entirety. Although there is no standard for percent agreement needed for consensus, previous studies suggest that agreement $\geq 80 \%$ is appropriate. ${ }^{17}$ As such, we set the consensus at $\geq 80 \%$ participant agreement. This study was approved by the Research Ethics Board at St. Michael's Hospital, Toronto, Ontario.

\section{Statistical analysis}

Descriptive statistics and Cronbach's alpha for internal consistency were calculated using Microsoft Excel 2010 (Redmond, Washington).

\section{RESULTS}

Fourteen experts were recruited from six acute care specialties (see Table 1). All participants had performed a BAC in a simulation setting, $50 \%$ had performed a BAC on a patient, and $71 \%$ had performed a cricothyroidotomy (without use of a bougie) in a real clinical situation.

Three rounds of the Delphi process were performed, and consensus was achieved by the final round (Table 2). The response rate for each round was $100 \%$. 


\begin{tabular}{lc}
\hline Table 1. Expert demographics & \\
\hline Summary of 14 participants for Delphi process & Median (IQR) \\
\hline Years in practice & $19(9.5)$ \\
Number of BAC performed on task trainer & $45(40)$ \\
Number of cricothyroidotomies performed & $1(3.25)$ \\
in real life, per respondent & $0(1)$ \\
Number of BAC performed in clinical & \\
setting, per respondent & Number \\
\hline Clinical specialties of Delphi participants* & 10 \\
\hline Emergency & 3 \\
Anesthesia & 1 \\
General surgery & 2 \\
Critical care & 1 \\
Emergency medical services physician & 1 \\
Rural medicine & Number \\
\hline Country of practice of Delphi participants & 3 \\
\hline Australia & 8 \\
Canada & 3 \\
USA & \\
\hline *Four participants listed training multiple specialties. & \\
\hline
\end{tabular}

\section{Round 1}

The preliminary checklist contained 22 items. Four steps were combined with other steps, and one step was removed entirely while the remaining steps were unchanged. Step four initially stated that "BAC performance requires the clinician to stand on the patient's right side." Nine participants responded that handedness, available workspace and feasibility requires less prescriptive wording. In its initial state, the step received a mean ranking of 3.0. Participants indicated that it should remain in the checklist, however, with appropriate wording. Step four was revised to "if space allows, stand on the same side of your patient as your dominant hand." The term laryngeal handshake was replaced with a more accurate anatomic description to identify the cartilaginous complex comprising the hyoid, thyroid, and cricoid. Additionally, experts suggested a smaller incision from the thyroid cartilage to cricoid cartilage to avoid excess bleeding/trauma. A caveat was included that the incision can be extended to the sternal notch when anatomic landmarks are disrupted.

\section{Round 2}

The revised checklist following Round 1 modifications contained 17 items. After the second round, all steps had a mean score $>3.0$. No steps were removed or added; however, two steps were modified for clarity. Participants expressed concern that including "prepare the neck with chlorhexidine swabs" may lead to unnecessary delays in prioritizing sterility instead of actual procedure performance. This step was modified to "if time allows, clean neck with sterilizing solution or swabs," and it remained in the final iteration of the checklist. Experts also advocated that end-tidal $\mathrm{CO}_{2}$ monitors should be attached to the endotracheal tube for immediate confirmation of tube placement. These suggestions were included in the final iteration of the checklist. In this round, the Cronbach's alpha score was calculated to be 0.855 (95\% confidence interval 0.73 0.94), demonstrating a very good degree of internal consistency.

\section{Round 3}

Given that no steps were removed or added in Round 2, the final round required experts to provide final approval of the 17-item checklist. During this round, a previously unmentioned concern was raised by several experts regarding bougie hold-up, that is, inserting the bougie into the trachea until it can no longer be advanced as a means to confirm airway and not esophageal location. As a result, participants were asked about the importance and necessity of inserting the bougie until hold-up. Some felt that this was a necessary step to help confirm bougie location within the airway. Others thought this step was unnecessary and dangerous because hold-up may still occur in an extra-tracheal soft tissue location. All experts agreed that a reliable technique to confirm the proper location of the bougie within the trachea was required. Based on participant suggestions, the checklist was modified to include digital palpation of the tracheal rings prior to bougie insertion. Following these modifications, each individual participant was electronically sent the checklist for review and asked to indicate whether they agreed on the final iteration. Eighty-six percent of the experts concluded that this 17-item checklist was acceptable for instruction of BAC to novices (Box 1).

\section{DISCUSSION}

This study is the first Delphi-derived checklist for BAC, a novel cricothyroidotomy technique. This represents essential progress towards the development 
Checklist item

1. Identify necessary materials for BAC: gum-elastic bougie, number 10 blade scalpel, size 6 ETT tube, 10cc syringe.

2. Provide supraglottic oxygenation.

3. Load ETT over bougie.

4. Stand on the right side of patient.

5. Palpate thyroid eminence at midline of neck.

6. Identify space between thyroid eminence and cricoid cartilage.

7. Prepare neck with chlorhexidine swabs.

8. Landmark using the laryngeal "handshake."

9. Hold number 10 blade in right hand.

10. Make a vertical incision through skin, aiming caudally from thyroid cartilage to sternal notch at midline of neck.

11. Palpate cricothyroid membrane.

12. Make a transverse incision through cricothyroid membrane.

13. Insert left index finger into transverse incision.

14. Pick up bougie with right hand.

15. Slide the coude tip of the bougie beside left index finger into incision space.

16. Railroad ETT over bougie until cuff is no longer visible above cricothyroid membrane.

17. Remove bougie.

18. Inflate cuff.

19. Attach ETT to BVM.

20. Assess for appropriate tube placement: auscultate both lungs, end tidal $\mathrm{CO} 2$ measurement.

21. Secure the tube.

22. Confirm tube placement with X-ray.

\section{Round 1}

Mean; median

(standard deviation) Result (standard deviation) Result Result

\begin{tabular}{|c|c|c|c|c|}
\hline $6.8 ; 7(0.6)$ & WC & $6.3 ; 7(0.9)$ & - & - \\
\hline $6.5 ; 7(1.0)$ & - & $6.2 ; 7(0.9)$ & - & - \\
\hline $2.8 ; 2$ (1.9) & * & & & \\
\hline $3 ; 3(1.7)$ & WC & $4.9 ; 5$ (1.4) & - & - \\
\hline $4.7 ; 5(2.5)$ & * & & & \\
\hline $6 ; 7(2.2)$ & WC & $4.5 ; 5$ (1.9) & - & - \\
\hline $3.7 ; 4(1.9)$ & - & $4.1 ; 4(1.8)$ & WC & - \\
\hline $4.6 ; 4$ (1.9) & WC & $5.7 ; 6(1.1)$ & - & - \\
\hline $4.5 ; 5$ (2.3) & * & & & \\
\hline $5.4 ; 6(2.0)$ & WC & $6 ; 6$ (1.33) & - & WC \\
\hline $5.9 ; 7$ (1.7) & WC & $6.6 ; 7(0.8)$ & - & WC \\
\hline $6.3 ; 7(1.2)$ & - & $6.6 ; 7(1.0)$ & - & - \\
\hline $5 ; 6$ (1.9) & WC & $6.4 ; 6(0.9)$ & - & - \\
\hline $4.8 ; 4(2.1)$ & * & & & \\
\hline $5.7 ; 6(1.4)$ & WC & $6.5 ; 6(0.8)$ & - & - \\
\hline $6.5 ; 7$ (1.0) & - & $6.9 ; 7(0.7)$ & - & - \\
\hline $6.2 ; 7(1.7)$ & - & $6.9 ; 7$ (1.2) & - & - \\
\hline $6.3 ; 7$ (1.7) & - & $6.9 ; 7$ (1.2) & - & - \\
\hline $6 ; 7$ (2.3) & - & $6.9 ; 7$ (1.2) & WC & - \\
\hline 7; 7 (0) & WC & $7.1 ; 7$ (1.2) & - & - \\
\hline $6.5 ; 7(1.0)$ & - & $7 ; 6.5(1.1)$ & - & - \\
\hline $4.9 ; 6(2.0)$ & * & & & \\
\hline
\end{tabular}

* Removed, or combined with another step.

of a validated checklist for the instruction and assessment of performance of BAC. Although task-specific checklists should not be used exclusively in lieu of global rating scales for assessment, they represent an important training tool to standardize instruction, particularly during the early stage of skill acquisition. ${ }^{19}$ Task-specific checklists in procedural skills training facilitate learners' exposure to predefined steps necessary for success, especially where clinical opportunities are so rare that real-time training is not possible and simulation-based training represents a viable alternative. ${ }^{11,12,20}$

In this study, we established consensus using the combined experience of an international panel of experts. In essence, the sum of experience is greater than the experience of each individual. By soliciting and collating feedback from multiple experts, participants were able to review, reflect on, and revise each step anonymously with a broader perspective than had it been a solitary exercise. Furthermore, the rarity of this procedure is such that even experts are relatively inexperienced, in contrast to experts in endotracheal intubation or central venous catheter placement. The benefit of Delphi methodology was highlighted in the final round when several experts independently raised concerns regarding bougie hold-up and the potential for the false confirmation of bougie location. While it is likely most had not considered this pitfall, especially if they had not experienced it themselves, the majority agreed that this may lead to patient harm. As such, the step was revised, and bougie hold-up was omitted. 


\section{Box 1. Final Checklist Items}

Steps to performing a Bougie-assisted Cricothyroidotomy

1. Identify necessary materials for BAC: bougie, scalpel, size 6 ETT, larger ETT options, 10cc syringe

2. Provide supraglottic oxygenation, if time permits

3. If space allows, position yourself on the same side of the patient as your dominant hand (Right hand dominant, stand on Right side patient)

4. Palpate up from sternal notch to find cricoid cartilage, and palpate down from mandible to identify the superior margin of thyroid cartilage. Palpate for the cricothyroid membrane (CTM)

5. If time allows, clean neck with sterilizing solution or swabs

6. With your non-dominant hand, stabilize the larynx using thumb and middle finger and palpate CTM with index finger.

7. With scalpel in dominant hand, make a vertical incision from thyroid cartilage to bottom of cricoid cartilage. If no palpable anatomy, extend incision toward sternal notch

8. Palpate through incision to confirm CTM

9. Extend CTM incision laterally in both directions without removing blade

10. Remove blade \& insert non-dominant index finger into transverse incision confirming with palpation of tracheal rings

11. With dominant hand, slide bougie into incision space

12. Railroad ETT over bougie until cuff is no longer visible above cricothyroid membrane

13. Remove bougie

14. Inflate Cuff

15. Attach ETT to ET CO2 and then BVM to confirm tube placement

16. Assess for appropriate tube placement: auscultate both lungs, $X$-ray if time allows

17. Secure the tube

The value of Delphi-derived checklists for procedural skill education is well described. Multiple studies have developed task-specific checklists for procedural skills to improve upon traditional steps that lacked systematic consideration of skill performance. ${ }^{11-16}$ This study suggests that the BAC is no exception. The first iteration of the BAC checklist was based on the existing literature and aligned with the steps proposed by the DAS. However, none of those checklists were established using any systematic consensus technique, and so individuals in these groups are vulnerable to be swayed by group dynamics, group think, and social psychology. ${ }^{21}$ In fact, once subject to expert review and Delphi methodology, the first iteration of the checklist underwent considerable modification. The Delphi process requires each individual to consider each item for themselves, informed by the previous round but not directly influenced by the opinions of others in the group. The benefit of this process is that multiple perspectives are included, with a safeguard against group decision-making and inherent social biases. ${ }^{17} \mathrm{We}$ propose that this checklist, derived from the Delphi method, is a more robust checklist than ones created by more traditional group consensus models because it not only involved specialists from diverse backgrounds and experiences, but also it allowed each voice in the group to be weighted equally; in the Delphi method, one voice does not overpower that of another.

There are three notable differences between this checklist and other published BAC guidelines. Firstly, the current DAS guidelines recommend standing on the opposite side of the patient as the handedness of the clinician (i.e., patient left for right-handed clinician). This creates awkward positioning to create a caudad incision because the clinician cannot easily stabilize the hand holding the scalpel on the patient's sternum. In contrast, study participants agreed that the clinician should (when possible) be positioned on the same side of the patient as the hand holding of the scalpel (i.e., patient right for right-handed clinician), to facilitate continuous stabilization of the larynx with the nondominant hand while the clinician makes a vertical incision using the dominant hand. Another description of the BAC technique advises only use of a horizontal incision through the skin and cricothyroid membrane. ${ }^{22}$ While this obviates the need for two incisions (vertical through skin followed by horizontal through the cricoid membrane), this represents a potential pitfall when landmark identification is inaccurate.

Secondly, the term laryngeal handshake, coined by Dr. Rich Levitan, is recommended in the DAS guidelines. ${ }^{1}$ This technique requires the clinician to palpate the entire hyoid-thyroid-cricoid complex to better appreciate patient anatomy. Study participants agreed, however, that this term is inherently vague and requires context and specific instruction. Although it may be useful for experienced clinicians, it was deemed to be confusing for novices.

Thirdly, the confirmation of correct bougie placement within the trachea was deemed critical by the expert panel. As mentioned previously, controversy emerged when bougie hold-up was originally suggested as a means to confirm tracheal placement. The DAS guidelines, however, do not provide guidance for this 
step. ${ }^{1}$ Participants agreed that digital palpation of tracheal rings was superior to hold-up to confirm proper location within the trachea.

\section{LIMITATIONS}

This study has several limitations. It included 14 experts from multiple specialities and institutions providing a broad perspective across a range of care models; however, we recognize that, although efforts were made to include an expansive sample of experts from around the world, an inherent limitation of our snowball sampling method was that our expert group did not include representation from all continents. It did, however, include experts from across Canada, the United States, and Australia with a wide range of clinical expertise. Notably absent from the sample is representation by otolaryngology. We contacted two otolaryngologists, but they did not participate in the study. There remains considerable variability in the Delphi technique regarding the 1) definition of consensus, 2) method of expert selection, and 3) number of experts deemed adequate. ${ }^{11-17}$ Although we achieved our pre-specified target for agreement $(>80 \%)$, it was also not $100 \%$, speaking to inherent variability in BAC practice. Finally, although the initial checklist was derived from literature, the Delphi process also involves expert opinion only.

This checklist is intended to guide the instruction of novice learners in BAC performance. It should be regarded as a consensus-based attempt to standardize the training of a rare but life-saving procedure. However, it should not be considered the definitive technique for this skill. Clinicians work in a variety of settings that may affect their optimal positioning and performance ergonomics. For example, several prehospital clinicians commented that they may not always be able to position themselves on a preferred side of the patient, as suggested by the DAS guidelines, and that it is not always feasible or advisable to switch the scalpel from one hand to another during a procedure. ${ }^{1}$ Rather, our checklist represents an ideal state for performance, explicitly describing the steps of performing a BAC that allows domain or context-specific modification. Although this checklist may initially seem longer than other proposed BAC methods, we purposefully included every step as a means to provide educators and instructors with the most robust approach for novice education. Furthermore, inclusion of even the most seemingly simple and intuitive steps provided Delphi experts with an opportunity to feedback on practices that we may have otherwise not considered. Novice learners, however, should be first taught to correctly perform each step, and only after the skill is mastered and pitfalls are properly understood can shortcuts be introduced. This checklist has not been tested on learners and as such requires further validation as a tool for instructing novices. Further validity checks with simulation and real-time patients are necessary next steps in the development of this checklist.

\section{CONCLUSION}

In this study, we developed a 17-item, Delphi-derived, checklist to guide BAC instruction for novice learners the first systematically developed list of essential steps for a novel and critical airway procedure. This tool serves to standardize BAC skill instruction and provide learners with a structured and consistent set of steps for deliberate practice. Further studies are needed to understand the role of this checklist in assessing the performance of $\mathrm{BAC}$ and to validate it for BAC instruction.

Acknowledgement: The authors thank Andrea Meeson for her help in preparing this manuscript.

Competing interests: None declared.

\section{REFERENCES}

1. Frerk C, Mitchell VS, McNarry AF, et al. Difficult Airway Society 2015 guidelines for management of unanticipated difficult intubation in adults. Br 7 Anaesth 2015;115(6):827-48.

2. Reid LA, Dunn M, McKeown DW, Oglesby AJ. Surgical airway in emergency department intubation. Eur 7 Emerg Med 2011;18(3):168-71.

3. Peters J, Bruijstens L, van der Ploeg J, et al. Indications and results of emergency surgical airways performed by a physician-staffed helicopter emergency service. Injury 2015;46(5):787-90.

4. Michelson JD, Manning L. Competency assessment in simulation-based procedural education. Am 7 Surg 2008;196 (4):609-15.

5. Warner KJ, Sharar SR, Copass MK, Bulger EM. Prehospital management of the difficult airway: a prospective cohort study. 7 Emerg Med 2009;36(3):257-65.

6. McGaghie WC, Issenberg SB, Cohen ER, et al. Does simulation-based medical education with deliberate practice yield better results than traditional clinical education? A meta-analytic comparative review of the evidence. Acad Med 2011;86(6):706-11.

7. Quick JA, MacIntyre AD, Barnes SL. Emergent surgical airway: comparison of the three-step method 
and conventional cricothyroidotomy utilizing highfidelity simulation. 7 Emerg Med 2014;46(2):304-7.

8. Melchiors J, Todsen T, Nilsson P, et al. Preparing for emergency: a valid, reliable assessment tool for emergency cricothyroidotomy skills. Otolaryngol Head Neck Surg 2015;152(2):260-5.

9. Hill C, Reardon R, Joing S, et al. Cricothyrotomy technique using gum elastic bougie is faster than standard technique: a study of emergency medicine residents and medical students in an animal lab. Acad Emerg Med 2010;17 (6):666-9.

10. Lake FR, Hamdorf JM. Teaching on the run tips 5: teaching a skill. Med 7 Aust 2004;181(6):327-8.

11. Berg D, Berg K, Riesenberg LA, et al. The development of a validated checklist for thoracentesis: preliminary results. $\mathrm{Am}$ 7 Med Qual 2013;28(3):220-6.

12. Berg K, Riesenberg LA, Berg D, et al. The development of a validated checklist for radial arterial line placement: preliminary results. Am 7 Med Qual 2014;29(3):242-6.

13. Hartman N, Wittler M, Askew K, Manthey D. Delphi method validation of a procedural performance checklist for insertion of an ultrasound-guided internal jugular central line. Am 7 Med Qual 2016;31(1):81-5.

14. Ma IW, Pugh D, Mema B, et al. Use of an error-focused checklist to identify incompetence in lumbar puncture performances. Med Educ 2015;49(10):1004-15.
15. Maloney E, Dougherty P, Dighe M, Relyea-Chew A. The development of a validated checklist for ultrasoundguided thyroid nodule fine-needle aspiration biopsies: preliminary results. Ultrasound Q 2015;31(3):159-65.

16. Riesenberg LA, Berg K, Berg D, et al. The development of a validated checklist for femoral venous catheterization: preliminary results. Am 7 Med Qual 2014;29(5):445-50.

17. Hsu C-C, Sandford BA. The Delphi Technique: Making Sense of Consensus. Practical Assessment, Research and Evaluation 2007;12(10). Available at: http://pareonline.net/ getvn.asp?v=12\&n=10 (accessed 1 Sept 2016).

18. Kuzel A. Sampling in qualitative inquiry. In Crabtree BMW (ed.) Doing qualitative research. Thousand Oaks, CA: Sage Publications; 1999: 33-45.

19. Ilgen JS, Ma IW, Hatala R, Cook DA. A systematic review of validity evidence for checklists versus global rating scales in simulation-based assessment. Med Educ 2015;49(2):161-73.

20. Petrosoniak A, Herold J, Woolfrey K. Emergency medicine procedural skills: what are residents missing? CFEM 2013;15 (4):241-8.

21. Janis I. Groupthink: the desperate drive for consensus at any cost. In Shafritz J, Ott J, Jang Y (eds.) Classics of organization theory, 8th ed. Boston, MA: Cengage Learning; 2015.

22. Langvad S, Hyldmo PK, Nakstad AR, et al. Emergency cricothyrotomy - a systematic review. Scand 7 Trauma Resusc Emerg Med 2013;21:43. 